\title{
DESIGN OF A BUILDING FIRE PUMP SYSTEM WITH INTEGRATED PARALLEL PUMP
}

\author{
Rahmad Samosir ${ }^{1}$, Kimar Turnip ${ }^{2}$, Leonard Michael Odi Nathanael ${ }^{*}{ }^{\square}$ \\ 1, 2,*3 Mechanical Engineering Department, Universitas Kristen Indonesia, Jl. Mayjen Sutoyo No.2, \\ RT.9/RW.6, Cawang, Kec. Kramat jati, Kota Jakarta Timur, Daerah Khusus Ibukota Jakarta 13630, \\ Indonesia
}

DOI: https://doi.org/10.29121/granthaalayah.v9.i2.2021.3374

Article Type: Research Article

Article Citation: Rahmad Samosir, Kimar Turnip, and Leonard Michael Odi Nathanael. (2021). DESIGN OF A BUILDING FIRE PUMP SYSTEM WITH INTEGRATED PARALLEL PUMP. International Journal of Research -GRANTHAALAYAH, 9(2), 203-215.

https://doi.org/10.29121/granthaa layah.v9.i2.2021.3374

Received Date: 01 February 2021 Accepted Date: 28 February 2021

Keywords:

Design

Pump

Fire Extinguisher

Head Losses

Integrated

Hydrant

\section{ABSTRACT}

Multi-storey building must be equipped with a reliable building fire extinguishing system. The existing fire extinguishing system design is not equipped with a backup pump and a jockey pump. Based on The DKI Jakarta Governor Regulation No. 92/2014 and the Indonesian National Standard, to solve this problem, a building fire pump system is designed with a combination of the jockey pump, main pump and backup pump that connected in an integrated parallel arrangement. This design allows the backup pump to automatically run due to a pressure drop in the system pipe if the main pump fails or unable to build up pressure. When a fire occurs and a lot of hydrants are used, the backup pump will automatically run to help the main pump with a signal triggered by the pressure switch.

\section{INTRODUCTION}

Multi-storey building must be equipped with a fire extinguishing system. The building fire system must comply with applicable standards. The DKI Jakarta Governor Regulation No. 92/2014 is one of the standards related to the building fire pump system applicable in the DKI Jakarta area. Based on fire extinguishing standards, the main part of a building fire extinguisher is the pumping system in the pump room consisting of the main pump, backup pump, and runway pump.

The current fire extinguishing pump system design discussed in several previous journals has a weakness in the absence of a backup pump or it is equipped with a backup pump but is not integrated with the main pump. There is a risk of danger if the main pump fails to operate in a fire emergency. This paper discusses the solution of the existing problems by designing a building fire system using a main pump and a backup pump that are connected in parallel with an integrated running scenario in accordance with the DKI Jakarta Governor Regulation No. 92/2014 concerning Technical Requirements and Procedures for Installation of Pipe and Fire Hose Systems and Yard

(C) 2021 The Author(s). This is an open access article distributed under the terms of the Creative Commons Attribution License, which permits unrestricted use, distribution, and reproduction in any medium, provided the original author and source are credited. 
Hydrants and the Indonesian National Standard No. 03-1745-2000 concerning Procedures for Planning and Installation of Upright and Slang Pipe Systems for Prevention of Fire Hazards in Houses and Buildings .

The backup pump is designed to run automatically with a drop in system pressure detected by the pressure switch if the main pump fails. The backup pump is not only used when the main pump is in trouble or is in the maintenance process. However, when a fire occurs and many hydrant valves are opened, resulting in the main pump being unable to build up pressure, the backup pump will automatically run and help the main pump deliver water to the hydrant.

\section{AIM OF RESEARCH}

To produce a building fire pump system design with an integrated parallel pump series in accordance with the DKI Jakarta Governor Regulation No. 92/2014 and Indonesian National Standard No. 03-1745-2000.

\section{MATERIALS AND METHOD}

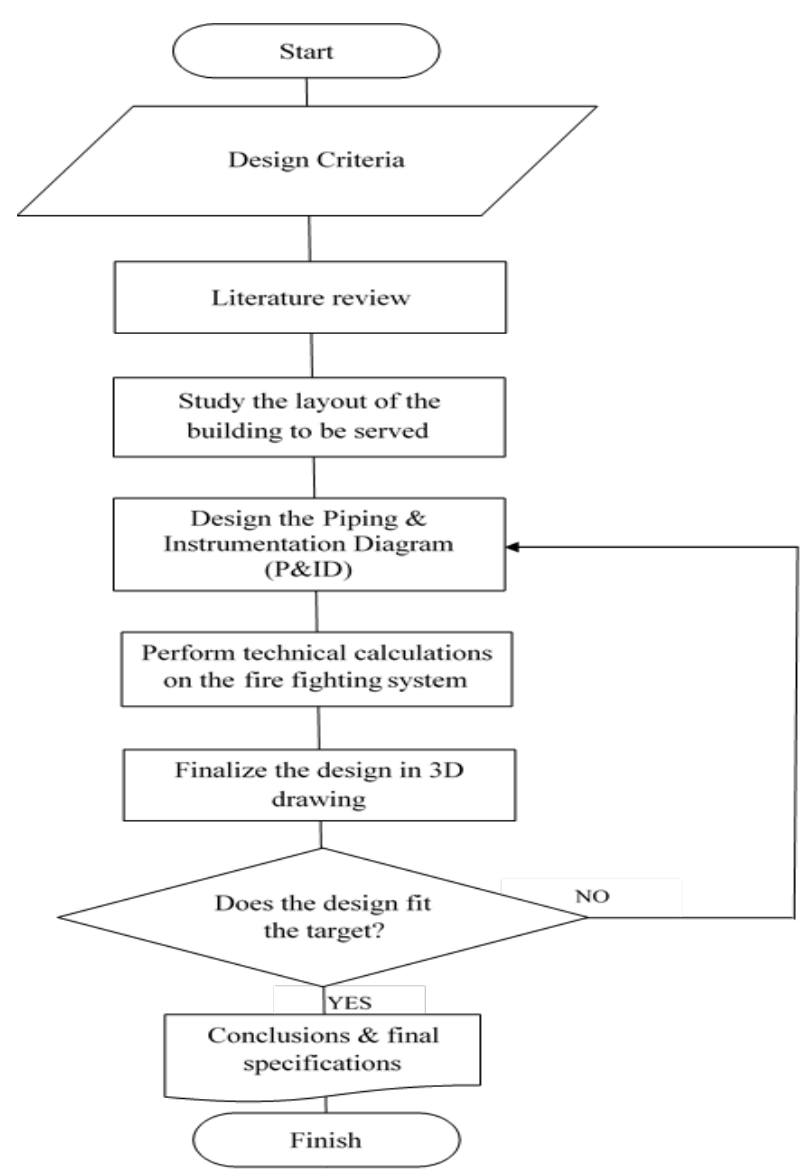

Figure 1: Flowchart of the Planning Process

\section{RESULTS AND DISCUSSIONS}

\subsection{BUILDING LAYOUT WITH HYDRANT PIPE INSTALLATION}

The building served by the damkar pump design has 7 floors with a height of $\pm 24[\mathrm{~m}]$. The building is equipped with 2 vertical distribution pipes. The output line for the building firefighting system is served only through the hydrant valve. The building hydrant pipe installation flow is described by a red line with the dimensions of the pipe $\emptyset 100$ [mm] according to the DKI Jakarta Governor Regulation No. 92/2014. 


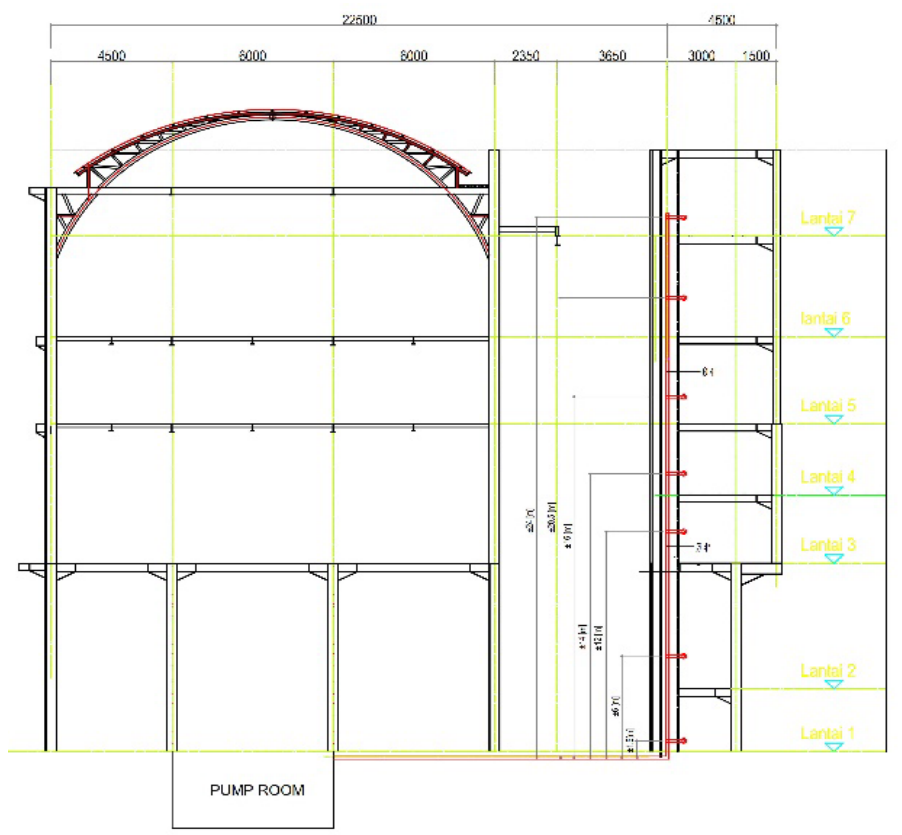

Figure 2: Front View of Building Layout with Hydrant Pipe Installation

\subsection{FIRE EXTINGUISHING SYSTEM TECHNICAL SPECIFICATIONS}

Based on the DKI Jakarta Governor Regulation No. 92/2014, for a multi-storey building with 2 vertical distribution pipes, the minimum flow rate in a standpipe must be 0,0315 [m3/s] (500 [usgpm]) and 0,0158 [m3/s] (250 [usgpm]) for the second standpipe. The hydrant provides a hose box with a DN $65[\mathrm{~mm}]$ nozzle. Then it can be seen the total flow rate in the vertical pipe according to the DKI Jakarta Governor Regulation No. 92/2014 is 0,0473 $[\mathrm{m} 3 / \mathrm{s}]$ (750 [usgpm]).

Based on the DKI Jakarta Governor Regulation No. 92/2014 can be seen the following data:

Nominal Diameter of Upright Pipe $\quad=100[\mathrm{~mm}]$

Nominal Diameter of Suction Pipe $\quad=150[\mathrm{~mm}]$

Nominal Diameter of Discharge Pipe $\quad=150[\mathrm{~mm}]$

Nominal Diameter of Header Pipe $\quad=150[\mathrm{~mm}]$

Minimum Safety Valve Size $\quad=100[\mathrm{~mm}]$

Based on the technical specifications that have been determined based on the DKI Jakarta Governor Regulation No. 92/2014, a piping \& instrumentation diagram (P\&ID) can be prepared as a reference frame for the design of a building fire pump system.

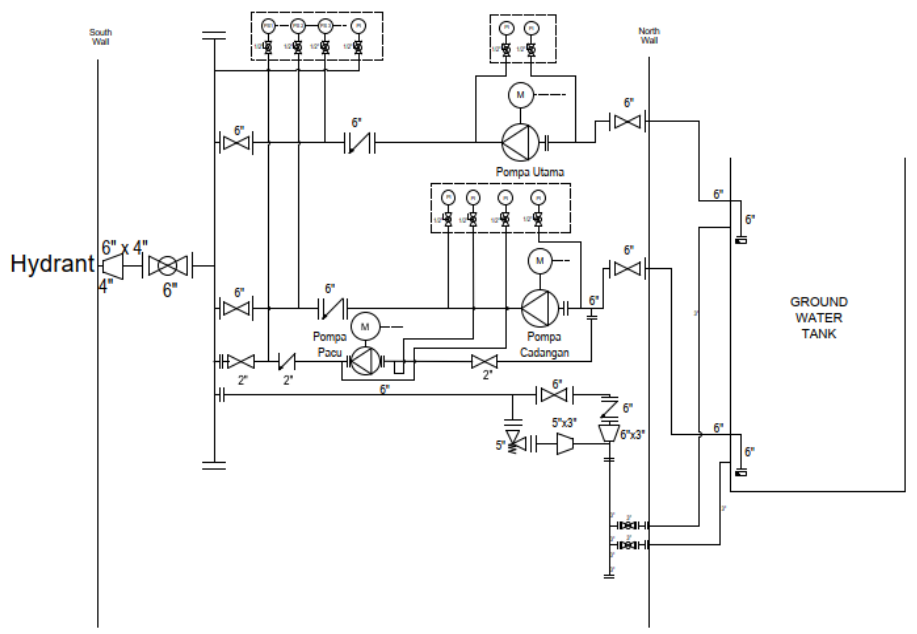

Figure 3: piping \& instrumentation diagram (P\&ID) 


\subsubsection{TECHNICAL SPECIFICATION OF SUCTION SPOOL PIPE}

Using carbon steel pipes:

$\begin{array}{lll}\rho_{\text {water }} & =995,65\left[\mathrm{~kg} / \mathrm{m}^{3}\right] \\ \mathrm{V} & & =1[\mathrm{cSt}]=0,000001\left[\mathrm{~m}^{2} / \mathrm{s}\right] \\ \mathrm{Q} & & =0,0473\left[\mathrm{~m}^{3} / \mathrm{s}\right] \\ \mathrm{g} & & =9,81\left[\mathrm{~m} / \mathrm{s}^{2}\right] \\ \text { Pipe roughness }(\varepsilon) & & =0,045[\mathrm{~mm}]=0,000045[\mathrm{~m}] \\ \text { ID pipe } \emptyset 150[\mathrm{~mm}] & & =0,154[\mathrm{~m}] \\ \text { L pipe } \varnothing 150[\mathrm{~mm}] & & =3[\mathrm{~m}] \\ \text { Elbow } \emptyset 150[\mathrm{~mm}] & & =1[\mathrm{pcs}] \\ \text { Strainer } \varnothing 150[\mathrm{~mm}] & & =1[\mathrm{pcs}] \\ \text { Gate valve } \varnothing 150[\mathrm{~mm}] & =1[\mathrm{pcs}] \\ \text { K elbow } \varnothing 150[\mathrm{~mm}] & =0,204 \\ \text { K strainer } \varnothing 150[\mathrm{~mm}] & =6 \\ \text { K gate valve } \varnothing 150[\mathrm{~mm}] & =0,2\end{array}$

\subsubsection{TECHNICAL SPECIFICATION OF DISCHARGE SPOOL PIPE}

\begin{tabular}{|c|c|}
\hline$\rho_{\text {water }}$ & $=995,65\left[\mathrm{~kg} / \mathrm{m}^{3}\right]$ \\
\hline $\mathrm{v}$ & $=1[\mathrm{cSt}]=0,000001\left[\mathrm{~m}^{2} / \mathrm{s}\right]$ \\
\hline g & $=9.81\left[\mathrm{~m} / \mathrm{s}^{2}\right]$ \\
\hline Q & $=0,0473\left[\mathrm{~m}^{3} / \mathrm{s}\right]$ \\
\hline Pipe roughness $(\varepsilon)$ & $=0,045[\mathrm{~mm}]=0,000045[\mathrm{~m}]$ \\
\hline ID pipe $\emptyset 150$ [mm] & $=0,154[\mathrm{~m}]$ \\
\hline L pipe $\varnothing 150[\mathrm{~mm}]$ & $=0,7[\mathrm{~m}]$ \\
\hline ID pipe $\varnothing 100$ [mm] & $=0,102[\mathrm{~m}]$ \\
\hline L pipe $\varnothing 100[\mathrm{~mm}] 1$ st floor & $=25,5[\mathrm{~m}]$ \\
\hline L pipe $\varnothing 100[\mathrm{~mm}]$ 2nd floor & $=30[\mathrm{~m}]$ \\
\hline L pipe $\emptyset 100[\mathrm{~mm}]$ 3rd floor & $=36[\mathrm{~m}]$ \\
\hline L pipe $\emptyset 100[\mathrm{~mm}]$ 4th floor & $=38[\mathrm{~m}]$ \\
\hline L pipe $\emptyset 100[\mathrm{~mm}]$ 5th floor & $=40[\mathrm{~m}]$ \\
\hline L pipe $\varnothing 100[\mathrm{~mm}] 6$ th floor & $=44.5[\mathrm{~m}]$ \\
\hline L pipe $\varnothing 100[\mathrm{~mm}] 7$ th floor & $=48[\mathrm{~m}]$ \\
\hline ID pipe $\varnothing 65$ [mm] & $=0,063[\mathrm{~m}]$ \\
\hline L pipe $\emptyset 65[\mathrm{~mm}]$ & $=0,3[\mathrm{~m}]$ \\
\hline Elbow $\emptyset 100[\mathrm{~mm}]$ & $=1[\mathrm{pcs}]$ \\
\hline Reducer $\emptyset 150 \times 100$ [mm] & $=1[\mathrm{pcs}]$ \\
\hline Check valve $\emptyset 150$ [mm] & $=1[\mathrm{pcs}]$ \\
\hline Globe valve $\varnothing 150$ [mm] & $=1[\mathrm{pcs}]$ \\
\hline Gate valve $\varnothing 150[\mathrm{~mm}]$ & $=1[\mathrm{pcs}]$ \\
\hline K reducer $\emptyset 150$ x100 [mm] & $=0,3$ \\
\hline K elbow $\emptyset 100[\mathrm{~mm}]$ & $=0,22$ \\
\hline K check valve $\emptyset 150$ [mm] & $=1,5$ \\
\hline K globe valve $\varnothing 150$ [mm] & $=3,5$ \\
\hline K gate valve $\varnothing 150[\mathrm{~mm}]$ & $=0,2$ \\
\hline
\end{tabular}




\subsection{TECHNICAL CALCULATION ANALYSIS ON SUCTION PIPE LINES}

\subsubsection{FINDING THE SURFACE AREA IN A PIPE (A)}

$$
\begin{aligned}
\mathrm{A} & =\frac{\pi}{4} \cdot \mathrm{d}^{2} \\
& =\frac{\pi}{4} \cdot(0,154[\mathrm{~m}])^{2} \\
& =0,0186\left[\mathrm{~m}^{2}\right]
\end{aligned}
$$

Remarks:

A $=$ the cross-sectional area in the pipe $\left[\mathrm{m}^{2}\right]$

$\mathrm{d}=$ pipe inner diameter $[\mathrm{m}]$

\subsubsection{DETERMINING THE REYNOLD NUMBER (RE)}

$\operatorname{Re} \quad=\frac{\text { u.d }}{\mathrm{v}}$

$$
\begin{aligned}
& =\frac{2,54[\mathrm{~m} / \mathrm{s}] \cdot 0,154[\mathrm{~m}]}{0,000001\left[\mathrm{~m}^{2} / \mathrm{s}\right]} \\
& =391204,23
\end{aligned}
$$

So, it can be obtained:

$$
\text { Re. } \begin{aligned}
\frac{\varepsilon}{\mathrm{d}} & =391204,23 \cdot \frac{0,000045[\mathrm{~m}]}{0,154[\mathrm{~m}]} \\
& =114,31
\end{aligned}
$$

Remarks:

$\mathrm{u} \quad=$ linear velocity of water flow $[\mathrm{m} / \mathrm{s}]$

$\mathrm{d}=\quad=$ pipe inner diameter $[\mathrm{m}]$

$\mathrm{v}=$ kinematic viscosity $\left[\mathrm{m}^{2} / \mathrm{s}\right]$

$\operatorname{Re} \quad=$ reynold number

$\varepsilon \quad=$ inner surface roughness of the pipe

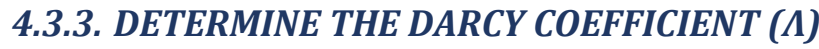

$\frac{1}{\sqrt{\lambda}} \quad=-2 \log _{10}\left(0,269 \frac{\varepsilon}{d}+\frac{2.51188}{\operatorname{Re} \sqrt{\lambda}}\right)$

Remarks

$\lambda=$ darcy coefficient

$\mathrm{d}=$ inner diameter of pipe [m]

$\varepsilon=$ inner surface roughness of the pipe

$\mathrm{Re} \quad=$ reynold number

To solve the above equation, the iteration method was calculated using Microsoft Excel@ software to obtain the convergent darcy coefficient $(\lambda)$. Based on the results of iteration calculations, the convergent value of the darcy coefficient is $\lambda=0,016$

\subsubsection{CALCULATE HEAD LOSS ON PIPE Ø150 [MM]}

$$
\begin{aligned}
\mathrm{hr} \quad & =8 \cdot \lambda \cdot\left(\frac{\mathrm{Q}}{\pi^{2} \cdot \mathrm{g} \cdot \mathrm{d}^{5}}\right) \ldots \ldots \ldots \ldots \ldots \ldots \ldots \ldots \ldots \ldots \ldots \ldots \ldots \ldots \ldots \ldots \ldots \ldots \ldots \ldots \ldots \ldots \ldots \ldots \ldots \ldots \ldots \ldots \ldots \\
& =8 \cdot 0,016 \cdot\left(\frac{0,0473\left[\mathrm{~m}^{3} / \mathrm{s}\right]}{\pi^{2} \cdot 9,81\left[\mathrm{~m} / \mathrm{s}^{2}\right] \cdot(0,154[\mathrm{~m}])^{5}}\right) \\
& =0,102[\mathrm{~m}]
\end{aligned}
$$


Remarks:

$\lambda=$ darcy coefficient

$\mathrm{Q} \quad=$ flow $\left[\mathrm{m}^{3} / \mathrm{s}\right]$

$\mathrm{g} \quad=$ gravity acceleration $\left[\mathrm{m} / \mathrm{s}^{2}\right]$

$\mathrm{d}=$ inner diameter of pipe $[\mathrm{m}]$

$\mathrm{hr} \quad=$ Head loss $[\mathrm{m}]$

\subsubsection{CALCULATE HEAD LOSS ON ELBOW Ø150 [MM]}

On the suction line there is 1 pcs elbow $\emptyset 150$ [mm] schedule 40 long radius, so the losses can be calculated by:

$\mathrm{hr}$

$$
\begin{aligned}
& =8 \cdot \mathrm{K} \cdot\left(\frac{\mathrm{Q}}{\pi^{2} \cdot \mathrm{g} \cdot \mathrm{d}^{4}}\right) \ldots \ldots \ldots \cdots \cdots \cdots \cdots \cdots \cdots \cdots \cdots \cdots \cdots \cdots \cdots \cdots \cdots \cdots \cdots \cdots \cdots \\
& =8 \cdot 0,204 \cdot\left(\frac{0,0473\left[\mathrm{~m}^{3} / \mathrm{s}\right]}{\pi^{2} \cdot 9,81\left[\mathrm{~m} / \mathrm{s}^{2}\right] \cdot(0,154[\mathrm{~m}])^{4}}\right) \\
& =0,067[\mathrm{~m}]
\end{aligned}
$$

Remarks:

$$
\begin{array}{ll}
\mathrm{Q} & =\text { flow }[\mathrm{m} 3 / \mathrm{s}] \\
\mathrm{g} & =\text { gravity acceleration }\left[\mathrm{m} / \mathrm{s}^{2}\right] \\
\mathrm{d} & =\text { inner diameter of pipe }[\mathrm{m}] \\
\mathrm{K} & =\text { loss coefficient } \\
\mathrm{hr} & =\text { Head loss }[\mathrm{m}]
\end{array}
$$

\subsubsection{CALCULATE HEAD LOSS ON GATE VALVE Ø150 [MM]}

In the inlet there is 1 pcs gate valve $\emptyset 150$ [mm] as required in the DKI Jakarta Governor Regulation No. 92/2014 and the losses can be calculated as much as:

$$
\begin{aligned}
\mathrm{hr} \quad & =8 \cdot \mathrm{K} \cdot\left(\frac{\mathrm{Q}}{\pi^{2} \cdot \mathrm{g} \cdot \mathrm{d}^{4}}\right) \ldots \ldots \ldots \ldots \ldots \ldots \ldots \ldots \ldots \ldots \ldots \ldots \ldots \ldots \ldots \ldots \ldots \ldots \ldots \ldots \\
& =8 \cdot 0,2 \cdot\left(\frac{0,0473\left[\mathrm{~m}^{3} / \mathrm{s}\right]}{\pi^{2} \cdot 9,81\left[\mathrm{~m} / \mathrm{s}^{2}\right] \cdot(0,154[\mathrm{~m}])^{4}}\right) \\
& =0,066[\mathrm{~m}]
\end{aligned}
$$

\subsubsection{CALCULATE HEAD LOSS ON SUCTION PIPELINE}

Total head loss in the main pump suction pipe line can be obtained by adding up all the head losses in the main pump suction pipe.

$$
\begin{aligned}
\text { hr total } & =\text { hr pipe }+ \text { hr elbow }+ \text { hr strainer }+ \text { hr gate valve } \\
& =0,203[\mathrm{~m}]+0,067[\mathrm{~m}]+1,973[\mathrm{~m}]+0,066[\mathrm{~m}] \\
& =2,208[\mathrm{~m}]
\end{aligned}
$$

\subsection{CALCULATE HEAD LOSS ON DISCHARGE PIPELINE}

\subsubsection{FINDING THE INNER SURFACE AREA ON PIPE (A)}

$$
\text { A } \quad=\frac{\pi}{4} \cdot \mathrm{d}^{2}
$$


Rahmad Samosir, Kimar Turnip, and Leonard Michael Odi Nathanael

Table 1: The result of the calculation of the inner surface area of the pipe

\begin{tabular}{|c|c|c|}
\hline No. & $\varnothing[\mathrm{mm}]$ & $\mathrm{A}\left[\mathrm{m}^{2}\right]$ \\
\hline 1 & 65 & 0,0031 \\
\hline 2 & 100 & 0,0082 \\
\hline 3 & 150 & 0,0186 \\
\hline
\end{tabular}

\subsubsection{DETERMINE THE LINEAR VELOCITY OF FLOW IN A PIPE (U)}

$\mathrm{u} \quad=\frac{\mathrm{Q}}{\mathrm{A}}$

Table 2: The results of the calculation of the linear velocity of flow in the pipe

\begin{tabular}{|c|c|c|}
\hline No. & $\varnothing[\mathrm{mm}]$ & $\mathrm{u}[\mathrm{m} / \mathrm{s}]$ \\
\hline 1 & 65 & 15,18 \\
\hline 2 & 100 & 5,79 \\
\hline 3 & 150 & 2,54 \\
\hline
\end{tabular}

\subsubsection{DETERMINE THE REYNOLD NUMBER (RE)}

$\operatorname{Re} \quad=\frac{\text { u.d }}{\mathbf{v}}$

Table 3: The results of the calculation of the reynold number

\begin{tabular}{|c|c|c|}
\hline No. & $\varnothing[\mathrm{mm}]$ & Re \\
\hline 1 & 65 & 956277 \\
\hline 2 & 100 & 590641,68 \\
\hline 3 & 150 & 391204,23 \\
\hline
\end{tabular}

\subsubsection{DETERMINE THE DARCY COEFFICIENT (ג)}

$\frac{1}{\sqrt{\lambda}}=-2 \log _{10}\left(0,269 \frac{\varepsilon}{d}+\frac{2.51188}{\operatorname{Re} \sqrt{\lambda}}\right)$

To solve the above equation, the iteration method was calculated using Microsoft Excel $®$ software to obtain the convergent darcy coefficient $(\lambda)$ for each pipe. Based on the results of the iteration calculations above, the convergent Darcy coefficient values can be obtained as follows:

Table 4: The results of the calculation of the darcy coefficient

\begin{tabular}{|c|c|c|}
\hline No. & $\varnothing[\mathrm{mm}]$ & $\lambda$ \\
\hline 1 & 65 & 0,0185 \\
\hline 2 & 100 & 0,0171 \\
\hline 3 & 150 & 0,0165 \\
\hline
\end{tabular}

\subsubsection{CALCULATE HEAD LOSS ON PIPE}

$\mathrm{hr}=8 \cdot \lambda \cdot \mathrm{L}\left(\frac{\mathrm{Q}^{2}}{\pi^{2} \cdot g \cdot \mathrm{d}^{5}}\right)$

Table 5: The results of the head loss calculation on the pipe

\begin{tabular}{|c|c|c|}
\hline No. & $\varnothing[\mathrm{mm}]$ & $\mathrm{h}_{\mathrm{r}}[\mathrm{m}]$ \\
\hline 1 & 65 & 1,035 \\
\hline 2 & $100\left(1^{\text {st }}\right.$ floor $)$ & 7,317 \\
\hline
\end{tabular}


Design of A Building Fire Pump System with Integrated Parallel Pump

\begin{tabular}{|c|c|c|}
\hline 3 & $100\left(^{\text {nd }}\right.$ floor $)$ & 8,609 \\
\hline 4 & $1003^{\text {rd }}$ floor $)$ & 10,33 \\
\hline 5 & $1004^{\text {th }}$ floor $)$ & 14,289 \\
\hline 6 & $100\left(^{\text {th }}\right.$ floor $)$ & 14,863 \\
\hline 7 & $1006^{\text {th }}$ floor $)$ & 16,154 \\
\hline 8 & $100\left(^{\text {th }}\right.$ floor $)$ & 17,159 \\
\hline 9 & 150 & 0,063 \\
\hline \multicolumn{3}{|c}{} \\
\hline
\end{tabular}

\subsubsection{CALCULATE HEAD LOSS ON REDUCER Ø150X100 [MM]}

In the discharge line of the pump there is 1 pcs reducer $\emptyset 150$ x 100 [mm], so the losses can be calculated by:

$$
\begin{aligned}
\mathrm{hr} & =8 \cdot \mathrm{K} \cdot\left(\frac{\mathrm{Q}}{\pi^{2} \cdot \mathrm{g} \cdot \mathrm{d}^{4}}\right) \ldots \ldots \ldots \ldots \ldots \ldots \ldots \ldots \ldots \ldots \ldots \ldots \ldots \ldots \ldots \ldots \ldots \ldots \ldots \\
& =8 \cdot 0,3 \cdot\left(\frac{0,0473\left[\mathrm{~m}^{3} / \mathrm{s}\right]}{\pi^{2} \cdot 9,81\left[\mathrm{~m} / \mathrm{s}^{2}\right] \cdot(0,128[\mathrm{~m}])^{4}}\right) \\
& =0,207[\mathrm{~m}]
\end{aligned}
$$

\subsubsection{CALCULATE HEAD LOSS ON ELBOW $\emptyset 100$ [MM]}

On the suction line there is 1 pcs elbow $\emptyset 100$ [mm] schedule 40 long radius, so the losses can be calculated as much as:

$$
\begin{aligned}
\mathrm{hr} \quad & =8 \cdot \mathrm{K} \cdot\left(\frac{\mathrm{Q}}{\pi^{2} \cdot \text { g.d }}\right) \ldots \ldots \ldots \ldots \ldots \ldots \ldots \ldots \ldots \ldots \ldots \ldots \ldots \ldots \ldots \ldots \ldots \ldots \ldots \ldots \\
& =8 \cdot 0,22 \cdot\left(\frac{0,0473\left[\mathrm{~m}^{3} / \mathrm{s}\right]}{\pi^{2} \cdot 9,81\left[\mathrm{~m} / \mathrm{s}^{2}\right] \cdot(0,102[\mathrm{~m}])^{4}}\right) \\
& =0,369[\mathrm{~m}]
\end{aligned}
$$

\subsubsection{CALCULATE HEAD LOSS ON CHECK VALVE Ø150 [MM]}

In the inlet there is 1 pcs check valve $\emptyset 150[\mathrm{~mm}]$ as required in the DKI Jakarta Governor Regulation No. $92 / 2014$ and the losses can be calculated as much as:

$$
\begin{aligned}
\mathrm{hr} \quad & =8 \cdot \mathrm{K} \cdot\left(\frac{\mathrm{Q}}{\pi^{2} \cdot \mathrm{g} \cdot \mathrm{d}^{4}}\right) \ldots \ldots \ldots \ldots \ldots \ldots \ldots \ldots \ldots \ldots \ldots \ldots \ldots \ldots \ldots \ldots \ldots \ldots \ldots \ldots \\
& =8 \cdot 1,5 \cdot\left(\frac{0,0473\left[\mathrm{~m}^{3} / \mathrm{s}\right]}{\pi^{2} \cdot 9,81\left[\mathrm{~m} / \mathrm{s}^{2}\right] \cdot(0,154[\mathrm{~m}])^{4}}\right) \\
& =0,493[\mathrm{~m}]
\end{aligned}
$$

\subsubsection{CALCULATE HEAD LOSS ON GLOBE VALVE Ø150 [MM]}

In the inlet there is 1 pcs globe valve $\varnothing 150[\mathrm{~mm}]$ and the losses can be calculated as:

$$
\begin{aligned}
\mathrm{hr} & =8 \cdot \mathrm{K} \cdot\left(\frac{\mathrm{Q}}{\pi^{2} \cdot \mathrm{g} \cdot \mathrm{d}^{4}}\right) \ldots \ldots \ldots \ldots \ldots \ldots \ldots \ldots \ldots \ldots \ldots \ldots \ldots \ldots \ldots \ldots \ldots \ldots \ldots \\
& =8 \cdot 0,2 \cdot\left(\frac{0,0473\left[\mathrm{~m}^{3} / \mathrm{s}\right]}{\pi^{2} \cdot 9,81\left[\mathrm{~m} / \mathrm{s}^{2}\right] \cdot(0,154[\mathrm{~m}])^{4}}\right) \\
& =1,151[\mathrm{~m}]
\end{aligned}
$$




\subsubsection{CALCULATE HEAD LOSS ON GATE VALVE Ø150 [MM]}

In the inlet there is 1 pcs gate valve $\emptyset 150$ [mm] as required in the DKI Jakarta Governor Regulation No. 92/2014 and the losses can be calculated as much as:

$\mathrm{hr} \quad=8 \cdot \mathrm{K} \cdot\left(\frac{\mathrm{Q}}{\pi^{2} \cdot \mathrm{g} \cdot \mathrm{d}^{4}}\right)$

$$
\begin{aligned}
& =8 \cdot 0,2 \cdot\left(\frac{0,0473\left[\mathrm{~m}^{3} / \mathrm{s}\right]}{\pi^{2} \cdot 9,81\left[\mathrm{~m} / \mathrm{s}^{2}\right] \cdot(0,154[\mathrm{~m}])^{4}}\right) \\
& =0,066[\mathrm{~m}]
\end{aligned}
$$

\subsubsection{CALCULATE TOTAL HEAD LOSS ON DISCHARGE PIPELINE}

The total head loss in the main pump discharge line can be obtained by adding up all the head losses in the main pump discharge pipeline.

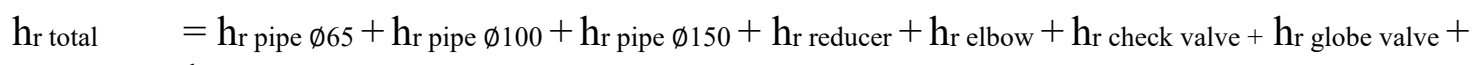
$h_{\text {r gate valve }}$

Based on the results of the above calculations, the total head loss can be obtained as follows:

Table 6: The results of the calculation of the total head loss on the discharge line

\begin{tabular}{|c|c|}
\hline Floor & $\mathrm{h}_{\text {total }}[\mathrm{m}]$ \\
\hline $1^{\text {st }}$ & 10,702 \\
\hline $2^{\text {nd }}$ & 11,993 \\
\hline $3^{\text {rd }}$ & 13,715 \\
\hline $4^{\text {th }}$ & 14,289 \\
\hline $5^{\text {th }}$ & 14,863 \\
\hline $6^{\text {th }}$ & 16,154 \\
\hline $7^{\text {th }}$ & 17,159 \\
\hline
\end{tabular}

Based on the DKI Jakarta Governor Regulation No. 92/2014, the minimum pressure at the highest hydrant point is $4,5[\mathrm{bar}]=450000[\mathrm{~Pa}]$, it can be determined that the minimum pump head required is:

$$
\begin{aligned}
\text { h pump } & =h_{\text {r total } 7 \text { th floor }}+\left(\frac{450000[\mathrm{~Pa}]}{\rho \cdot g}\right) \\
& =17,159[\mathrm{~m}]+\left(\frac{450000[\mathrm{~Pa}]}{995,65\left[\frac{\mathrm{kg}}{\mathrm{m}^{3}}\right] \cdot 9,81\left[\frac{\mathrm{m}}{\mathrm{s}^{2}}\right]}\right) \\
& =17,159[\mathrm{~m}]+46,07[\mathrm{~m}] \\
& =63,23[\mathrm{~m}] \approx 64[\mathrm{~m}]
\end{aligned}
$$

Then we can choose a pump with the following specifications:

$$
\begin{array}{ll}
\mathrm{Q} & =170,34\left[\mathrm{~m}^{3} / \mathrm{h}\right]=750[\mathrm{usgpm}] \\
\mathrm{H} & =64[\mathrm{~m}]
\end{array}
$$

\subsection{CALCULATE HYDRAULIC POWER}

$$
\begin{aligned}
\mathrm{Pw} & =\rho . \text { g. h. } \mathrm{Q} \\
& =995,65\left[\mathrm{~kg} / \mathrm{m}^{3}\right] \cdot 9,81\left[\mathrm{~m} / \mathrm{s}^{2}\right] \cdot 64[\mathrm{~m}] \cdot 0,047\left[\mathrm{~m}^{3} / \mathrm{s}\right] \\
& =29.380,12[\mathrm{~W}] \\
& =29,38[\mathrm{~kW}]
\end{aligned}
$$


Remarks:

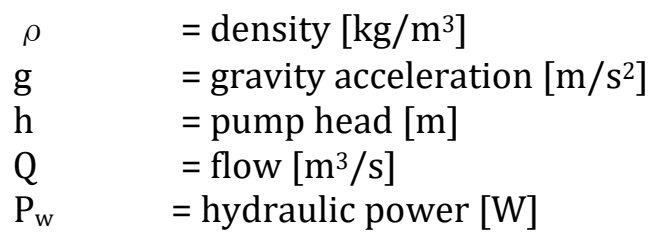

\subsection{CALCULATE ELECTRIC MOTOR POWER REQUIREMENTS}

It is assumed that the working efficiency of the pump is $\eta_{p}=80[\%]$

$$
\begin{aligned}
\mathrm{P} & =\frac{\mathrm{P}_{\mathrm{w}}}{\eta_{\mathrm{p}}} \ldots \ldots \ldots \ldots \ldots \ldots \ldots \ldots \ldots \ldots \ldots \ldots \ldots \ldots \ldots \ldots \ldots \ldots \ldots \ldots \ldots \ldots \ldots \ldots \ldots \ldots \ldots \ldots \ldots \ldots \ldots \ldots \ldots \ldots \ldots \ldots \ldots \\
& =\frac{29,38[\mathrm{~kW}]}{0,8} \\
& =36,73[\mathrm{~kW}]
\end{aligned}
$$

Remarks:

$$
\text { Pw = hydraulic power }[\mathrm{kW}]
$$

$\eta \mathrm{p} \quad$ = pump efficiency [\%]

Then obtained that the minimum requirement of electric motor power to drive the pump is 37 [kW].

\subsection{CALCULATE MINIMUM WATER RESERVOIR CAPACITY}

In accordance with the criteria specified in the DKI Jakarta Pergub No. 92/2014, the building fire pump must be able to pump water for at least 60 [minutes]. By using a pump with a capacity of 0,0473 [m³ $/ \mathrm{s}](750$ [usgpm]), the minimum water capacity in the tank is calculated:

$$
\begin{aligned}
\mathrm{Q} & =\frac{\mathbf{v}}{\mathbf{t}} \\
\mathrm{V} & =\mathrm{Q} \cdot \mathrm{t} \\
& =0,0473\left[\mathrm{~m}^{3} / \mathrm{s}\right] \cdot 3600[\mathrm{~s}] \\
& =170,28\left[\mathrm{~m}^{3}\right] \\
\text { Remarks: } & \\
\mathrm{Q} & =\text { flow }\left[\mathrm{m}^{3} / \mathrm{s}\right] \\
\mathrm{V} & =\text { volume of water reservoir }\left[\mathrm{m}^{3}\right] \\
\mathrm{t} & =\text { time }[\mathrm{s}]
\end{aligned}
$$

Based on the calculation results, it can be concluded that the minimum water volume for the damkar system in buildings is $170,28\left[\mathrm{~m}^{3}\right] \approx 171\left[\mathrm{~m}^{3}\right]$

\subsection{CALCULATE SPECIFIC SPEED OF PUMP}

$$
\begin{aligned}
\mathrm{n}_{\mathrm{s}} & =\mathrm{n} \cdot \frac{\mathrm{Q}^{0.5}}{\mathrm{H}^{0.75}} \cdots \cdots \cdots \cdots \cdots \cdots \cdots \cdots \cdots \cdots \cdots \cdots \cdots \cdots \cdots \cdots \cdots \cdots \cdots \cdots \cdots \cdots \cdots \cdots \cdots \cdots \cdots \cdots \cdots \cdots \cdots \cdots \cdots \cdots \cdots \cdots \\
& =3000[\mathrm{rpm}] \cdot \frac{0.05^{0.5}}{82^{0.75}} \\
& =29,65[\mathrm{rpm}]
\end{aligned}
$$

Remarks:

$$
\begin{array}{ll}
\mathrm{n} & =\text { driver's speed }[\mathrm{rpm}] \\
\mathrm{H} & =\text { pump head }[\mathrm{m}]
\end{array}
$$


$\begin{array}{ll}\mathrm{n}_{\mathrm{s}} & =\text { specific speed of pump }[\mathrm{rpm}] \\ \mathrm{Q} & =\text { flow }\left[\mathrm{m}^{3} / \mathrm{s}\right]\end{array}$

\subsection{CALCULATE THE REQUIRED NET POSITIVE SUCTION HEAD (NPSHR)}

It is known that ns $=23,95<100$, then based on Figure 3 . above, the cavitation coefficient $(\sigma)$ is 0,03 . So that it can be calculated the NPSHr value is
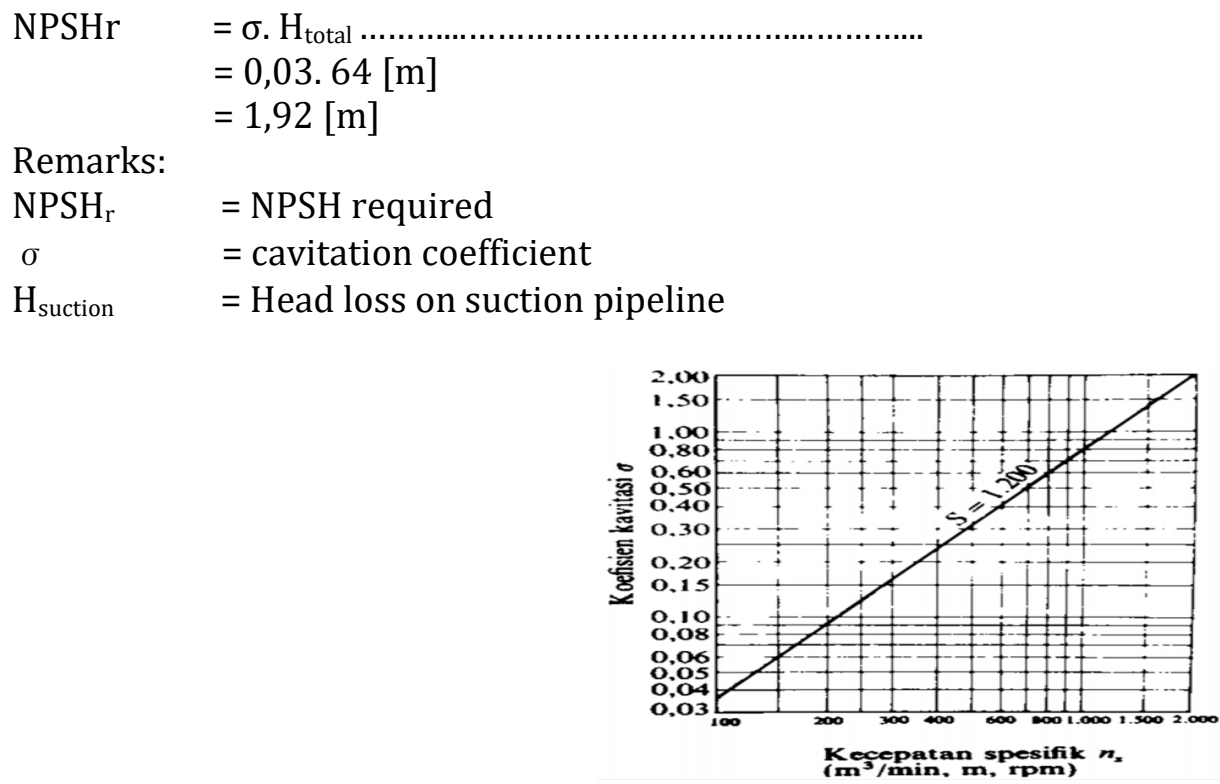

Figure 4: Graph of specific velocity and cavitation coefficient

(Source: Sularso, Tahara, (2000), Pompa dan Kompresor, Jakarta: PT Pradnya Paramita)

\subsection{CALCULATE THE MINIMUM WATER LEVEL IN THE RESERVOIR}

Net Positive Suction Head Available needs to be taken into account to keep fire pumps from the risk of cavitation. NPSHa is minimum so that the pump does not experience cavitation if NPSHa = NPSHr, so that the minimum water level in the tank to the pump centerline can be calculated as follows:

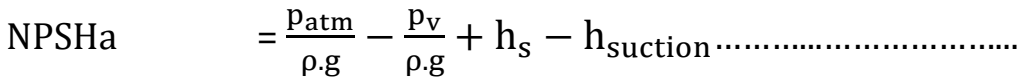

$$
\begin{aligned}
& \mathrm{h}_{\mathrm{s}} \quad=\mathrm{NPSHa}-\frac{\mathrm{p}_{\mathrm{atm}}}{\rho . \mathrm{g}}+\frac{\mathrm{p}_{\mathrm{v}}}{\rho . \mathrm{g}}+\mathrm{h}_{\text {suction }} \\
& =1,92[\mathrm{~m}]-\frac{101359,61[\mathrm{~Pa}]}{995,65\left[\frac{\mathrm{kg}}{\mathrm{m}^{3}}\right] \cdot 9,81\left[\frac{\mathrm{m}}{\mathrm{s}^{2}}\right]}+\frac{4240[\mathrm{~Pa}]}{995,65\left[\frac{\mathrm{kg}}{\mathrm{m}^{3}}\right] \cdot 9,81\left[\frac{\mathrm{m}}{\mathrm{s}^{2}}\right]}+2,208[\mathrm{~m}] \\
& =-5,82[\mathrm{~m}]
\end{aligned}
$$

Remarks:

$\mathrm{p}_{\mathrm{atm}} \quad=$ atmosphere pressure $[\mathrm{Pa}]$

$\rho \quad \quad \quad=$ fluid density $\left[\mathrm{kg} / \mathrm{m}^{3}\right]$

g = gravity acceleration $\left[\mathrm{m} / \mathrm{s}^{2}\right]$

hs $\quad=$ the static level of the pump against the water level $[\mathrm{m}]$

hr = head loss of the pipeline [m]

So that the pump does not experience cavitation, the water level in the tank must not be more than 5.82 [m] below the pump centerline. 


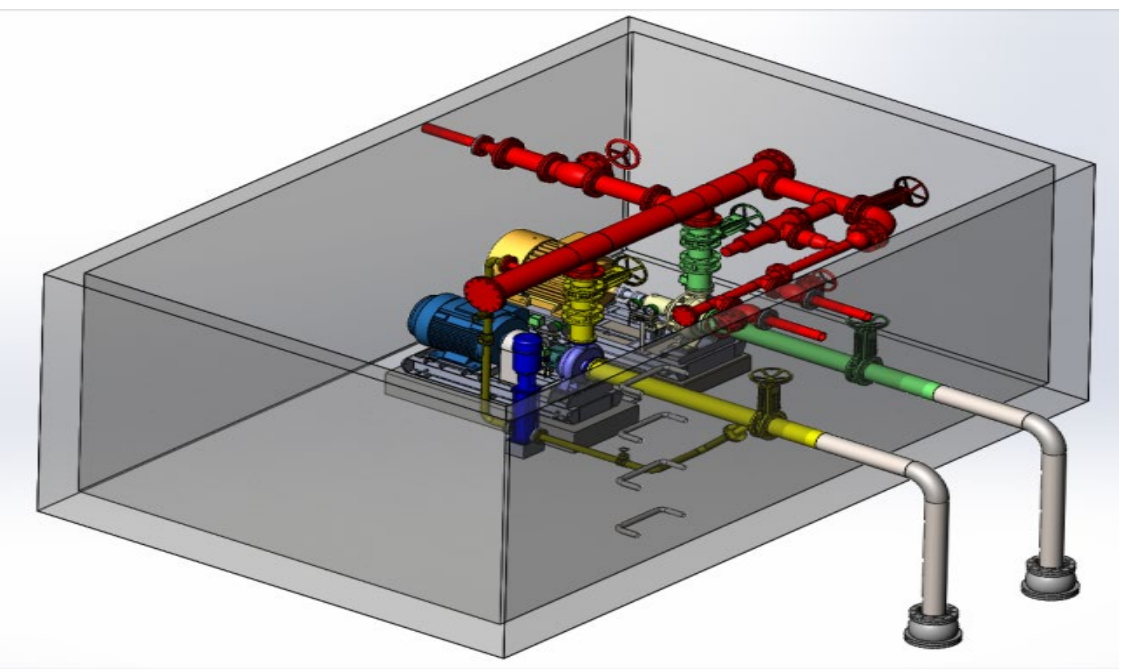

Figure 5: A sketch of the design of the fire pump in the pump room

\section{CONCLUSIONS AND RECOMMENDATIONS}

\subsection{CONCLUSIONS}

1) The building fire pump can be designed in accordance with the DKI Jakarta Governor Regulation No. 92/2014 and the Indonesian National Standard (SNI) with an integrated parallel pump. The backup pump not only functions as a substitute when the main pump fails or has a problem, but when a fire occurs and many hydrants are opened, the backup pump will also automatically run with a significant pressure drop in the damkar system. The main pump and the backup pump will run automatically when a pressure drop is triggered by the pressure switch.

2) The design specifications are as follows:

- The selected pump has a minimum head of $64[\mathrm{~m}]$

- $\quad$ Pump flow of $170,34\left[\mathrm{~m}^{3} / \mathrm{h}\right]$

- $\quad$ The required pump drive power is $37[\mathrm{~kW}]$

- Net Positive Suction Head required for a pump of 1,92 [m]

- The minimum water level in the tank to prevent cavitation from the pump should not be lower than 5,82 $[\mathrm{m}]$ below the pump centerline.

- The minimum capacity of the building firefighting water source tank is $171\left[\mathrm{~m}^{3}\right]$.

\subsection{RECOMMENDATION}

1) The design or modification of the building's fire pump needs to ensure the availability of the components and materials needed and on the market.

2) The design or modification of the building's fire pump needs to pay attention to the ease of the maintenance process for each of its main components.

\section{SOURCES OF FUNDING}

This research received no specific grant from any funding agency in the public, commercial, or not-for-profit sectors.

\section{CONFLICT OF INTEREST}

The author have declared that no competing interests exist. 


\section{ACKNOWLEDGMENT}

None.

\section{REFERENCES}

[1] Arifin, A.S. dkk, Analisis Instalasi Pompa Pemadam Kebakaran pada Kompleks Terminal Bahan Bakar Minyak Merauke, Universitas Musamus, 2014.

[2] Bosoni, Marco, dkk. Continuous Mixing Device, Particulary Suitable for Preparing Aqueous Solutions of Foam Extinguisher for Fire Fighting Systems.

[3] Darma, B.F. dkk., Evaluasi Fire Protection System pada Fuel Supply System, Utility Work Menggunakan Software Pipe Flow Expert (Study Kasus PT.Pertamina DPPU Juanda), Institut Teknologi Sepuluh November, Surabaya, 2014.

[4] Gunawan, Analisa Performansi Fire Pump DEP-0131-A dengan Standar NFPA 20 pada Lapangan South Processing Unit, Universitas Merdeka Malang, Malang, 2018.

[5] Haramain, M. A. dkk., Perancangan Sistem Pemadam Kebakaran pada Perkantoran dan Pabrik Label Makanan PT XYZ dengan Luas Bangunan 1125 [m2], Universitas Muhammadiyah Jakarta, Jakarta, 2017.

[6] Kurniadi, Rachmad, Perencanaan Sistem Plambing Dan Sistem Fire Hydrant Di Gedung Tower "A" Apartemen Bersubsidi Puncak Permai Surabaya, Institut Sepuluh November, Surabaya.

[7] Nabhan, Aziz Rafif. Priyonggo, Projek. dkk, Perencanaan Fire Fighting Menggunakan Fluida Air dan Busa di Gedung CPC Perusahaan Minyak Nabati, Politeknik Perkapalan Negeri Surabaya, Surabaya.

[8] Pergub DKI Jakarta No. 92 Tahun 2014, Persyaratan Teknis dan Tata Cara Pemasangan Sistem Pipa Tegak dan Slang Kebakaran serta Hidran Halaman.

[9] Putri, Rahesa Dwi, Perencanaan dan Analisa Sistem Sprinkler Otomatis dan Kebutuhan Air Pemadaman Fire Fighting Hotel XX, Universitas Mercu Buana, Jakarta, 2017.

[10] Rennels, Donald C., Pipe Flow, John Wiley \& Sons Inc, New Jersey, 2012.

[11] Sartono, R. P. H., Evaluasi Kinerja Pompa Pemadam Kebakaran Pada Gedung Bertingkat Berdasarkan Standar Peraturan SNI 03-6570-2001 dan NFPA 20, Universitas Negeri Jakarta, Jakarta.

[12] Siahaan, Uras. Kuslianto, Karyadi. Samosir, Rahmad., Pengembangan Sistem Pembangunan Kawasan Pasang Surut dan Pemanfaatan Sumber Daya Terbarukan dari Pompa Hempasan Gelombang Laut, Universitas Kristen Indonesia, Jakarta, 2015.

[13] Standar Nasional Indonesia 03-1745-2000, Tata Cara Perencanaan dan Pemasangan Sistem Pipa Tegak dan Slang untuk Pencegahan Bahaya Kebakaran pada Bangunan Rumah dan Gedung.

[14] Sularso, Tahara, Pompa dan Kompresor, PT Pradnya Paramita, Jakarta, 2000. 